\title{
Cardiovascular risk factors in the normoglycaemic Asian-Indian population-influence of urbanisation
}

\author{
C. Snehalatha $\cdot$ A. Ramachandran
}

Received: 27 September 2008 / Accepted: 13 January 2009/Published online: 10 February 2009

(C) Springer-Verlag 2009

\begin{abstract}
Aims/hypothesis The aim of this study was to investigate the prevalence of cardiovascular risk factors in normoglycaemic Asian-Indians and its association with urbanisation and plasma glucose.

Methods Data on 5,517 normoglycaemic individuals aged $\geq 20$ years $(1,521,1,851$ and 2,145 inhabitants of a city, a town and villages, respectively), from a 2006 diabetes survey, were used. The presence of single and multiple abnormalities, including obesity, abdominal obesity, hypertension and dyslipidaemia, was analysed. Risk associations for hypertension and dyslipidaemia were analysed using multivariate analysis after adjusting for confounding variables.

Results Both the prevalence and clustering of cardiovascular risk variables were higher in urban areas than in villages, and were higher in the city than in the town. Nearly half (47\%) of the normoglycaemic individuals from villages had at least one risk factor. Age, male sex, BMI, waist circumference, lack of physical activity and living in an urban environment were associated with hypertension and dyslipidaemia; postprandial glucose level showed no association with hypertension or dyslipidaemia after adjusting for these risk factors. Fasting plasma glucose level showed an independent association with dyslipidaemia. Conclusions/interpretation There is a high prevalence of cardiovascular risk factors even among normoglycaemic individuals, and this is highest in urban areas. Fasting
\end{abstract}

C. Snehalatha $\cdot$ A. Ramachandran $(\bowtie)$

India Diabetes Research Foundation,

Dr A. Ramachandran's Diabetes Hospitals,

28 Marshall's Road, Egmore,

Chennai 600 008, India

e-mail: ramachandran@vsnl.com plasma glucose level showed an association with dyslipidaemia but not hypertension.

Keywords Asian-Indian · Cardiovascular risk . Dyslipidaemia $\cdot$ Hypertension · Plasma glucose . Urbanisation

\section{Introduction}

The Indian subcontinent is one of the regions of the world with the highest burden of diabetes [1] and cardiovascular disease [2]. Cardiovascular disease occurs at a younger age in Indians compared with other populations [3]. The huge burden of cardiovascular disease in India and other countries of this subcontinent is attributed to a high prevalence of the risk factors, particularly diabetes. Diabetes and cardiovascular disease share several common aetiological factors, and India has the largest number of diabetic individuals in the world [1]. The prevalence of diabetes is rapidly increasing among the urban $[4,5]$ and urbanising rural populations in India [4]. Cardiovascular risk increases with increasing blood glucose levels [6]. Recently, we noted a high prevalence of cardiovascular risk factors in southern India, and this increased with the degree of urbanisation [4]. The objective of this analysis was to study the prevalence of cardiovascular risk factors in normoglycaemic individuals and to investigate whether this is correlated with urbanisation and blood glucose level.

\section{Methods}

Normoglycaemic individuals were selected from the database of a diabetes survey conducted by us in 2006 in a city, 
a town and villages [4]. In brief, an epidemiological survey of diabetes and prediabetic states (impaired glucose tolerance and impaired fasting glucose) was performed in the city of Chennai, Kanchipuram town, and villages of Panruti (19 villages randomly selected out of 42) in Tamil Nadu, India. The study was approved by the institutional ethics committee and informed consent was obtained from all study participants. Using a multistage random selection method a total of 7,066 adults aged $\geq 20$ years were surveyed (city $n=2,192$, town $n=2,290$ and villages $n=2,584$ ). Demographic and anthropometric details, together with information on medical history, education, monthly income, occupation, physical activity and tobacco use, were collected by personal interview. Height without shoes (in $\mathrm{cm}$ ), weight in light clothing (in $\mathrm{kg}$ ) and waist circumference (in $\mathrm{cm}$ ) were measured; BMI was calculated. Blood pressure at the upper arm was measured with a cufftype sphygmomanometer and the average of two readings was recorded. Physical activity at work, during leisure time and completing household activities was recorded as minutes of activity per week and divided into quartiles (sedentary, light, moderate and heavy levels).

Fasting and $2 \mathrm{~h}$ plasma glucose (with a $75 \mathrm{~g}$ anhydrous glucose load) were estimated in participants with no history of diabetes. Fasting serum total cholesterol and triacylglycerol were determined using enzymatic procedures, HDLcholesterol was determined by direct assay and the total cholesterol:HDL-cholesterol ratio was calculated (Roche Diagnostics, Mannheim, Germany). The glucose level of plasma samples in a fluoride tube was assayed by the glucose oxidase-peroxidase method using a Hitachi autoanalyser 912 (Roche Diagnostics). The coefficient of variation for this assay was $<2 \%$. Normoglycaemia was defined as a fasting plasma glucose level of $<6.1 \mathrm{mmol} / \mathrm{l}$ and a $2 \mathrm{~h}$ plasma glucose level of $<7.8 \mathrm{mmol} / \mathrm{l}$ [7]. Individuals with any form of glucose intolerance or known diabetes were excluded. Therefore, among the 7,066 individuals screened, only 5,517 qualified for this analysis.

Obesity (BMI $\geq 25 \mathrm{~kg} / \mathrm{m}^{2}$ ) and abdominal obesity (waist circumference of $\geq 90$ and $\geq 85 \mathrm{~cm}$ for men and women, respectively), were assessed. Hypertension was diagnosed if an individual had a systolic $\mathrm{BP} \geq 130 \mathrm{mmHg}$ or diastolic BP $\geq 85 \mathrm{mmHg}$ or if antihypertensive drugs were being used. Dyslipidaemia was defined by the presence of one or more of the following: total cholesterol $\geq 5.2 \mathrm{mmol} / 1$, triacylglycerol $\geq 1.7 \mathrm{mmol} / \mathrm{l}, \mathrm{HDL}$-cholesterol $<1.03 \mathrm{mmol} / \mathrm{l}$ and total cholesterol:HDL-cholesterol ratio of $>4.5$.

\section{Statistical analysis}

The prevalence of the different abnormalities was calculated as a percentage. Comparisons between the areas were made using $Z$ statistics. Mean values were compared using the Student's unpaired $t$ test. Multiple logistic regression analyses were used to determine the risk variables associated with hypertension or dyslipidaemia. Independent variables (categorised) included were age (10 year intervals), sex, BMI ( $5 \mathrm{~kg} / \mathrm{m}^{2}$ intervals), waist circumference (10 cm intervals), monthly income (2,500 rupee intervals), occupation, education and physical activity. A logistic regression model was used to measure the individual adjusted odds ratios for hypertension and dyslipidaemia in quintiles of fasting and $2 \mathrm{~h}$ glucose using the first quintile values as the reference values. Odds ratios were adjusted for age, sex, BMI, waist circumference and physical activity in the hypertension model and adjusted for age, sex, BMI and waist circumference in the dyslipidaemia model. A $p$ value of $<0.05$ was considered significant. SPSS for Windows (version 10.0; SPSS, Chicago, IL, USA) was used for analysis.

\section{Results}

The characteristics of the study participants $(n=5,517)$ and prevalence of risk variables are shown in Table 1. Mean age was higher in rural populations. The village dwellers had lower values for anthropometry, biochemical variables and blood pressure than urban participants (city or town). The prevalence of hypertension was higher in the city than in other areas (Table 1). There was a low prevalence of dyslipidaemia in the villages. Although the concentration of HDL-cholesterol was lower in the villages, the total cholesterol:HDL-cholesterol ratio was normal in a larger percentage of participants in this group than in the other two groups. Participants from the city were most likely to be in the sedentary and light physical activity categories (67\%), followed by the town (63\%) and the villages (51\%) (city vs town $z=2.4, p=0.017$; city vs villages $z=9.6$, $p<0.0001)$. Cigarette smoking was more common in the town and villages than in the city (city $11.9 \%$, town $22.8 \%$, villages $23.9 \%$ ); there were very few female smokers (data not shown). A positive family history of cardiovascular disease was reported by $7.2 \%, 2.5 \%$ and $1.0 \%$ of participants from the city, town and villages, respectively.

The prevalence of risk factors was higher in the city $(67.5 \%)$ than in the other two areas (city vs town $z=2.33$, $p=0.02$; city vs villages $z=12.27, p<0.0001$ ) (Fig. 1), and was higher in the town $(63.6 \%)$ than in the villages (47\%) $(z=10.48, p<0.0001)$. Multiple abnormalities, particularly combinations of three or more variables, were more common in urban areas, with a prevalence of $23.3 \%$, $17.6 \%$ and $10.4 \%$ in the city, town and villages, respectively (villages vs town $z=6.55$, town vs city $z=4.06$, villages vs city $z=10.52, p<0.001)$. 
Table 1 Anthropometric, haemodynamic and biochemical characteristics and prevalence of abnormalities in the study groups

\begin{tabular}{|c|c|c|c|c|c|c|}
\hline \multirow[t]{2}{*}{ Variable } & \multicolumn{3}{|l|}{ Measurement } & \multicolumn{3}{|c|}{ Abnormal measurement } \\
\hline & City & Town & Villages & City & Town & Village \\
\hline$n$, total (men, women) & $1,521(722,799)$ & $1,851(791,1,060)$ & $2,145(1,054,1,091)$ & - & - & - \\
\hline Age (years) & $34.9 \pm 11.5$ & $34.4 \pm 10.6$ & $37.6 \pm 11.6^{\mathrm{a}}$ & - & - & - \\
\hline BMI $\left(\mathrm{kg} / \mathrm{m}^{2}\right)$ & $23.6 \pm 4.8$ & $23.2 \pm 4.7$ & $21.1 \pm 4.0^{\mathrm{a}}$ & $534(35.1)$ & $592(32.0)$ & $367(17.1)^{\mathrm{a}}$ \\
\hline Waist circumference $(\mathrm{cm})$ & $81.9 \pm 11.0$ & $80.5 \pm 10.9$ & $76.9 \pm 9.8^{\mathrm{a}}$ & $617(40.6)$ & $706(38.1)$ & $492(22.9)^{\mathrm{a}}$ \\
\hline Systolic BP (mmHg) & $119.7 \pm 12.1$ & $118.1 \pm 12.3$ & $117.1 \pm 11.5^{\mathrm{b}}$ & - & - & - \\
\hline Diastolic BP (mmHg) & $75.9 \pm 8.9$ & $76.3 \pm 7.9$ & $74.8 \pm 8.2^{\mathrm{a}}$ & - & - & - \\
\hline Hypertension & - & - & - & $229(15.1)$ & $209(11.3)^{\mathrm{e}}$ & $170(7.9)^{\mathrm{a}}$ \\
\hline Cholesterol (mmol/l) & $4.47 \pm 0.94$ & $4.18 \pm 0.89^{\mathrm{c}}$ & $3.93 \pm 0.93^{\mathrm{a}}$ & $307(20.2)$ & $237(12.8)^{\mathrm{c}}$ & $186(8.7)^{\mathrm{a}}$ \\
\hline Triacylglycerol (mmol/l) & $1.39 \pm 0.85$ & $1.41 \pm 0.83$ & $1.33 \pm 0.89^{\mathrm{d}, \mathrm{e}}$ & $346(22.7)$ & $440(23.8)$ & $403(18.8)^{b}$ \\
\hline HDL-cholesterol (mmol/l) & $1.17 \pm 0.26$ & $1.13 \pm 0.25^{\mathrm{c}}$ & $1.09 \pm 0.24^{\mathrm{a}}$ & $466(30.6)$ & $650(35.1)^{\mathrm{e}}$ & $867(40.4)^{\mathrm{a}}$ \\
\hline Total cholesterol:HDL-cholesterol & $4.0 \pm 1.1$ & $3.8 \pm 1.0^{\mathrm{c}}$ & $3.7 \pm 0.94^{\mathrm{a}}$ & $388(25.5)$ & $382(20.6)^{\mathrm{c}}$ & $364(17.0)^{\mathrm{b}}$ \\
\hline
\end{tabular}

Data are presented as mean $\pm \mathrm{SD}$ or $n(\%)$ unless stated otherwise

${ }^{\mathrm{a}} p<0.0001$ vs city and town; ${ }^{\mathrm{b}} p<0.01$ vs city and town; ${ }^{\mathrm{c}} p<0.0001$ vs city; ${ }^{\mathrm{d}} p<0.01$ vs town; ${ }^{\mathrm{e}} p<0.05$ vs city

Age, male sex, BMI, waist circumference, being in the sedentary physical activity category and living in an urban environment were independently associated with hypertension. After adjusting for these variables, fasting and $2 \mathrm{~h}$ glucose values showed no association with hypertension. Dyslipidaemia showed an independent association with fasting glucose between the levels of 5.0 and $5.3 \mathrm{mmol} / \mathrm{l}$ (OR 1.162, 95\% CI 1.023-1.321, $p=0.021$ ).

\section{Discussion}

Normoglycaemic Asian-Indians have a high prevalence of cardiovascular risk factors, and this is influenced by urbanisation. Even among the rural participants, nearly $50 \%$ had at least one cardiovascular risk factor other than tobacco use. General obesity, upper body adiposity,

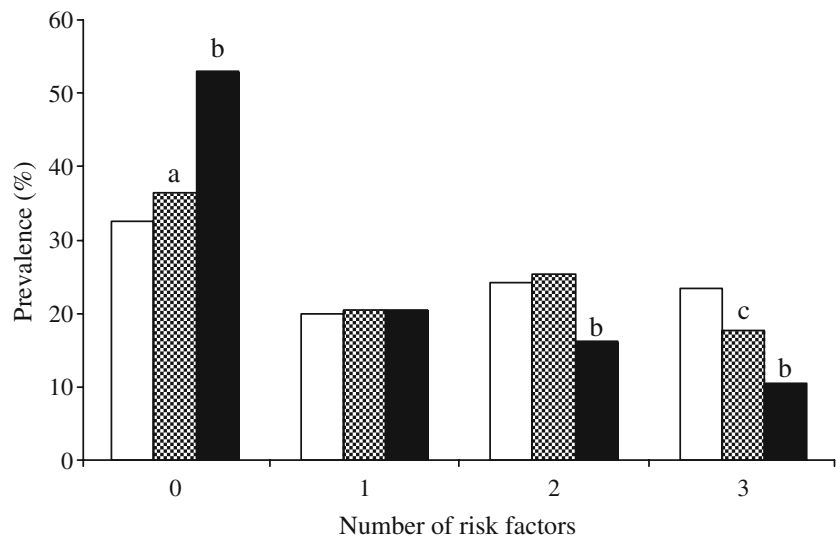

Fig. 1 Prevalence of single and multiple cardiovascular risk factors (as a percentage) in the city (white bars), town (grey bars) and villages (black bars). ${ }^{\mathrm{a}} p=0.02$ vs city, ${ }^{\mathrm{b}} p<0.001 \mathrm{vs}$ city and town, ${ }^{\mathrm{c}} p<0.0001$ vs city hypertension and dyslipidaemia were more common among the urban (city and town) residents than the rural participants. Abdominal obesity was the most common abnormality in the different groups. Several epidemiological studies in native Asian-Indians [4, 8] and migrant Indians [9] have shown a higher occurrence of upper body adiposity than general adiposity (BMI).

The ratio of total cholesterol:HDL-cholesterol was a better index of the dyslipidaemic status than was either variable considered separately, and was abnormal in a larger percentage of urban than rural participants. The lower HDL-cholesterol levels observed in villagers were largely due to lower total cholesterol values. Hypertriglyceridaemia was common in all groups. Upper body adiposity and hypertriglyceridaemia are proxy indices of insulin resistance $[9,10]$.

While the occurrence of single risk factors was similar in all groups, the presence of multiple risk factors was significantly lower in the rural population. The lower rates of obesity, particularly abdominal obesity, among the rural participants may explain this.

Urbanisation involves changes in lifestyle variables that promote cardiovascular risk factors. Among the normoglycaemic participants, glucose had no independent association with hypertension, while fasting glucose showed a weak association with dyslipidaemia.

A comparative study of non-diabetic healthy AsianIndians vs whites in America indicated that the Indians had multiple abnormalities; specifically, insulin resistance, dyslipidaemia, elevated plasminogen activator inhibitor I levels, impaired insulin-mediated vasodilatation and altered body fat distribution [9].

The results of this study suggest that conventional cardiovascular risk factors are present even in rural individuals and that the burden is increased in an urban 
environment. The increasing trends in diabetes and other dysglycaemic conditions are likely to increase the number of individuals at cardiovascular risk by many fold in the future.

Acknowledgements Funded by the India Diabetes Research Foundation.

Duality of interest The authors declare that there is no duality of interest associated with this manuscript.

\section{References}

1. Sicree R, Shaw J, Zimmet P (2006) Diabetes and impaired glucose tolerance-prevalence and projections. In: Gan D (ed) Diabetes atlas, 3rd edn. International Diabetes Federation, Brussels, pp 15-103

2. Goyal A, Yusuf A (2006) The burden of cardiovascular disease in the Indian subcontinent. Indian J Med Res 124:235-244
3. Ghaffar A, Reddy KS, Singhi M (2004) Burden of noncommunicable diseases in South Asia. BMJ 328:807-810

4. Ramachandran A, Mary S, Yamuna A, Murugesan N, Snehalatha $S$ (2008) High prevalence of diabetes and cardiovascular risk factors associated with urbanization in India. Diabetes Care 31:893-898

5. Gupta R, Misra A (2007) Type-2 diabetes in India: regional disparities. Br J Diabetes Vasc Dis 7:12-16

6. Gerstein HC, Pais P, Pogue J, Yusuf S (1999) Relationship of glucose and insulin levels to the risk of myocardial infarction: a case-control study. J Am Coll Cardiol 33:612-619

7. WHO (1999) Definition, diagnosis and classification of diabetes mellitus and its complications. Report of a WHO consultation. Part 1: diagnosis and classification of diabetes mellitus. WHO, Geneva

8. Shelgikar KM, Hockaday TDR, Yajnik CS (1991) Central rather than generalized obesity is related to hyperglycaemia in Asian Indian subjects. Diabet Med 8:712-717

9. Raji A, Seely EW, Arky RA, Simonson DC (2001) Body fat distribution and insulin resistance in healthy Asian Indians and Caucasians. J Clin Endocrinol Metab 86:5366-5371

10. Snehalatha C, Satyavani K, Sivasankari S, Vijay V (2005) Serum triglycerides as a marker of insulin resistance in non-diabetic urban Indians. Diab Res Clin Pract 69:205-206 